\title{
Radiographic evaluation of congruency of the first metatarsophalangeal joint in hallux valgus
}

Yan Li, Xu Tao* (10) and Kanglai Tang*

\begin{abstract}
Background: Congruency of the first metatarsophalangeal (MTP) joint is extremely important for the selection of surgical methods and prognosis, while radiographic evaluation methods are relatively lacking. The purpose of this article was to explore radiographic indicators for evaluating congruency of the first MTP joint.

Methods: We selected patients with hallux valgus who had a weightbearing X-ray in the outpatient system and measured their hallux valgus angle (HVA). In total, 183 cases of 245 feet with HVA greater than $15^{\circ}$ were selected. The distal metatarsal articular angle (DMAA), metatarsophalangeal joint angle (MTPJA), congruency index (Cl) and tibial sesamoid position (TSP) were measured and statistically analysed.
\end{abstract}

Results: The higher the degree of hallux valgus was, the higher the proportion of incongruency of the first MTP joint. Significant differences were found in the DMAA, MTPJA and $\mathrm{Cl}$ between the congruency and incongruency groups of patients with moderate-to-severe hallux valgus $(P<0.05)$. The areas under the curve (AUCs) of the receiver operating characteristic (ROC) curve for DMAA was $0.554(P>0.05)$. However, the MTPJA and $\mathrm{Cl}$ were 0.906 and 0.884 , the sensitivity values reached 0.791 and 0.949 , the specificity values were 0.862 and 0.644 , and the critical values were 10.67 and 0.765 , respectively. The correlation test indicated that in the congruency group, the DMAA and HVA were positively correlated, but the MTPJA, Cl and HVA had low correlation coefficients. The DMAA and HVA were not correlated in the incongruency group; however, the MTPJA and HVA were significantly positively correlated, and the $\mathrm{Cl}$ and HVA showed a negative correlation $(P<0.05)$.

Conclusion: The MTPJA and $\mathrm{Cl}$ are indicators that can be used to quantitatively evaluate the congruency of the first MTP joint, taking $10^{\circ}$ and 0.765 as the demarcation points, respectively. Clinically, congruency of the MTP joint should be considered when choosing surgical methods for different degrees of hallux valgus, and the MTPJA and CI can be used as quantitative evaluation indicators. Level of evidence: Level III, Retrospective Comparative Study.

Keywords: Hallux valgus; Metatarsophalangeal joint; Congruency; Metatarsophalangeal joint angle; Congruency index

*Correspondence: taoux@hotmail.com; tangkanglai@hotmail.com Department of Orthopedics/Sports Medicine Center, The First Affiliated Hospital of Army Medical University (Southwest Hospital of Gaotanyan Zhengjie), Shapingba District, Chongqing 400038, China

\section{Background}

Congruency of the first metatarsophalangeal (MTP) joint is important for the choice of hallux valgus surgery and post-operative recurrence [1]. However, the data on the congruency of the MTP joint are quite different $[2,3]$. Incongruency of the MTP joint affects a higher original author(s) and the source, provide a link to the Creative Commons licence, and indicate if changes were made. The images or other third party material in this article are included in the article's Creative Commons licence, unless indicated otherwise in a credit line to the material. If material is not included in the article's Creative Commons licence and your intended use is not permitted by statutory regulation or exceeds the permitted use, you will need to obtain permission directly from the copyright holder. To view a copy of this licence, visit http://creativecommons.org/licenses/by/4.0/. The Creative Commons Public Domain Dedication waiver (http://creativeco mmons.org/publicdomain/zero/1.0/) applies to the data made available in this article, unless otherwise stated in a credit line to the data. 
proportion of patients with moderate-to-severe hallux valgus. For patients with hallux valgus of the same degree, there are large differences in the choice of surgical methods due to the existence of MTP joint congruency and incongruency. For example, double metatarsal osteotomy (DMO) is often required for patients who have a large hallux valgus angle (HVA) and congruency of the MTP joint $[4,5]$. For patients with moderate-to-severe hallux valgus with incongruency metatarsophalangeal joints, scarf osteotomy or simple Chevron osteotomy is effective $[6,7]$.The previous literature used mostly the congruency or incongruency metatarsophalangeal joint as an index to evaluate the effect of hallux valgus surgery [8-10], and its measurement rests only on the arc ratio of the articular surface; however, there is no quantitative measurement index for the congruency of the first MTP joint [11].

The purpose of this article was to quantitatively evaluate the congruency of the MTP joint through two innovative indicators and conduct diagnostic experimental analysis to explore the imaging indicators of MTP joint, aiming to assess the evaluation value of the two new indicators and the intrinsic relationship between different MTP joint imaging indicators. The results will provide a basis for surgical selection of patients with different MTP joints and add quantitative indicators to evaluate MTP joints for post-operative follow-up.

\section{Methods}

Patients who had a weightbearing X-ray in the outpatient system from January 2018 to January 2021 were selected. HVA was measured in a PACS system. The inclusion criteria were age $\geq 18$ years; patients who underwent footbearing X-ray examination in the outpatient system; and patients who measured $\mathrm{HVA}>15^{\circ}$. The exclusion criteria were patients aged $<18$ years; patients with a history of foot and ankle trauma or fracture; or patients with a history of previous foot or ankle surgery. Finally, 183 hallux valgus patients with 245 feet were included, comprising 51 males and 132 females. Among the patients, 131 feet (53.47\%) had mild hallux valgus (HVA: $15-30^{\circ}$ ), 80 feet (32.65\%) had moderate hallux valgus (HVA: $31-40^{\circ}$ ), and 34 feet $(13.88 \%)$ had severe hallux valgus $\left(\mathrm{HVA} \geq 40^{\circ}\right)$ according to the classification criteria for hallux valgus [12].

The HVA, distal metatarsal articular angle (DMAA) and tibial sesamoid position (TSP) were measured in weightbearing foot anterior-posterior images according to the measurement method described in previous literature (Fig. 1A, B, E) [13]. According to the congruency and incongruency of the first MTP joint in patients with hallux valgus, we innovatively designed two new measurement indicators, the metatarsophalangeal joint angle (MTPJA) and congruency index (CI), which was defined by the MTPJA as follows: on the weightbearing foot anterior-posterior images, we drew a straight line between the inner and outer edges of the articular surface of the proximal phalanx and the distal metatarsal, respectively, and the angle between the two straight lines was the MTPJA (Fig. 1C). The CI was calculated as described in our previous article [6]. The ratio of the curve length of the metatarsophalangeal joint contact surface to the curve length of the metatarsal head cartilage surface was defined as the $\mathrm{CI}$, which was designed to measure the congruency of the MTP joint (Fig. 1D). There are currently no quantitative indicators to assess whether the metatarsophalangeal joint is congruent; it is based only on the physician's subjective observation of whether the articular surfaces at both ends of the metatarsophalangeal joint were parallel (Fig. 1F). Based on this, we selected two doctors with 20 years of experience in foot and ankle surgery and divided the 245 feet into the congruency and incongruency groups. The HVA, DMAA, MTPJA, CI and TSP were statistically analysed among different degrees of hallux valgus and between the congruency and incongruency groups. Diagnostic tests were performed for the DMAA, MTPJA and CI, and receiver operating characteristic (ROC) curves were plotted. The area under the curve (AUC), sensitivity, specificity and critical value were calculated.

SPSS 20.0 software was used for statistical analysis. Quantitative data were expressed as $\mathrm{X} \pm \mathrm{S}$. The categorical data were expressed as $n$ (\%). Comparisons of count data of patients were analysed by means of the Chi-square test. Comparisons of different parameters between patients with different degrees of hallux valgus were analysed by one-way analysis of variance, and the independent sample Student's $t$ test was used to compare different parameters between the congruency and incongruency groups. The diagnostic test of the DMAA, MTPJA and CI used ROC curves and calculated the AUC, critical value, sensitivity, specificity and other parameters. A correlation test was carried out between different parameters of the congruency and incongruency groups, and the correlation coefficient was calculated. The difference was considered to be statistically significant at $P<0.05$.

\section{Results}

The HVA, DMAA, MTPJA and TSP increased significantly as the degree of hallux valgus increased, while the $C I$ decreased $(P<0.05)$ (Table 1$)$. Using the previous observation of the parallelism of the first metatarsophalangeal joint surface as the gold standard [11], we divided 245 feet into the congruency and incongruency groups. In total, approximately $2 / 3$ (64.49\%) of the patients had first MTP joints that were congruent, and approximately $1 / 3(35.51 \%)$ of the patients had 


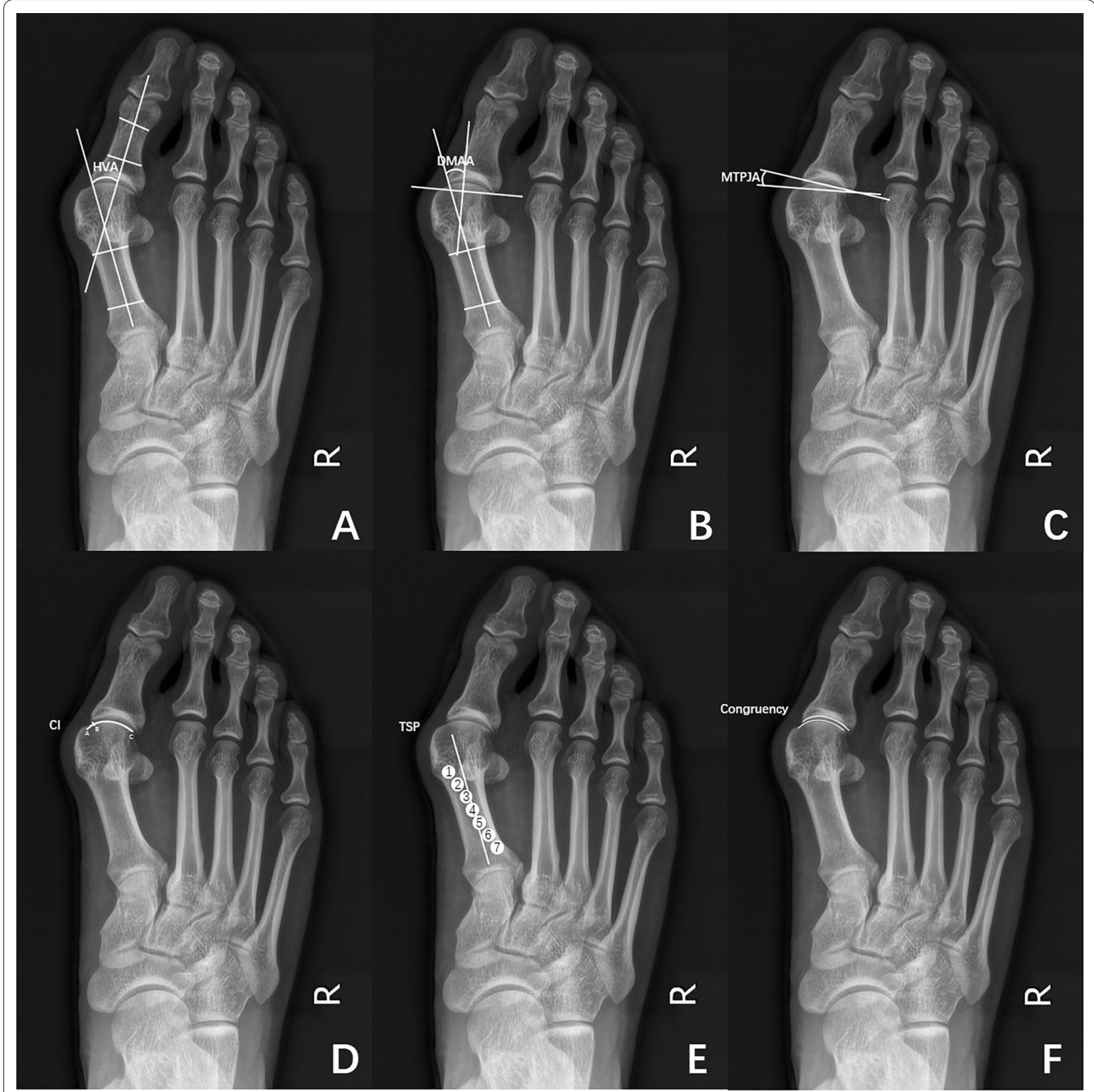

Fig. 1 The imaging indicators of the first MTP joint on the weightbearing foot anterior-posterior images. A hallux valgus angle, HVA; $\mathbf{B}$ distal metatarsal articular angle, DMAA; $\mathbf{C}$ metatarsophalangeal joint angle, MTPJA; D congruency index, Cl; E tibial sesamoid position, TSP; $\mathbf{F}$ evaluation of the congruency of the first MTP joint

first MTP joints that were incongruent. The majority of patients with mild hallux valgus have congruency, while those with severe hallux valgus have incongruency. Patients with moderate hallux valgus were basically the same. The differences among the three groups were statistically significant $(P<0.05)$ (Fig. 2$)$.

Although the difference in HVA between the two groups of patients with mild and moderate hallux valgus was statistically significant, the numerical differences were not significant. Patients with severe hallux valgus did not have a significant difference in HVA between the two groups. In the congruency group, the TSP of patients with mild to moderate hallux valgus was lower than that of the incongruency group, while there was no significant difference between the two groups of patients with severe hallux valgus. In terms of 
Table 1 Comparison and analysis of different indexes in patients with different degrees of hallux valgus

\begin{tabular}{|c|c|c|c|c|c|c|}
\hline Index & Mild hallux valgus & $P^{*}$ & Moderate hallux valgus & $P^{* *}$ & Severe hallux valgus & $P^{* * *}$ \\
\hline $\operatorname{Sex}(M / F)$ & $37 / 70$ & 0.060 & $15 / 55$ & 0.637 & $5 / 24$ & 0.073 \\
\hline Age (year) & $45.60 \pm 16.55$ & 0.064 & $51.44 \pm 17.33$ & 0.116 & $57.34 \pm 17.21$ & 0.003 \\
\hline$L / R$ & $69 / 62$ & 0.981 & $42 / 38$ & 0.807 & $17 / 17$ & 0.781 \\
\hline $\operatorname{HVA}\left({ }^{\circ}\right)$ & $24.07 \pm 4.01$ & 0.000 & $33.72 \pm 2.79$ & 0.000 & $46.52 \pm 4.71$ & 0.000 \\
\hline DMAA $\left({ }^{\circ}\right)$ & $12.55 \pm 5.78$ & 0.000 & $18.98 \pm 7.26$ & 0.040 & $22.01 \pm 10.75$ & 0.000 \\
\hline MTPJA $\left({ }^{\circ}\right)$ & $8.25 \pm 4.88$ & 0.000 & $12.91 \pm 7.45$ & 0.000 & $23.42 \pm 13.90$ & 0.000 \\
\hline $\mathrm{Cl}$ & $0.85 \pm 0.07$ & 0.000 & $0.80 \pm 0.09$ & 0.000 & $0.65 \pm 0.16$ & 0.000 \\
\hline TSP & $3.87 \pm 1.65$ & 0.000 & $5.15 \pm 1.67$ & 0.004 & $6.09 \pm 1.08$ & 0.000 \\
\hline Feet number (ratio) & $131(53.47 \%)$ & 0.003 & $80(32.65 \%)$ & 0.000 & $34(13.88 \%)$ & 0.000 \\
\hline Congruency/incongruency & $112 / 19$ & 0.000 & $38 / 42$ & 0.017 & $8 / 26$ & 0.000 \\
\hline
\end{tabular}

HVA hallux valgus angle, DMAA distal metatarsal articular angle, MTPJA the first metatarsophalangeal joint angle, Cl congruency index, TSP tibial sesamoid position $P^{*}$ : comparisons between mild and moderate hallux valgus. $P^{* *}$ : comparisons between moderate and severe hallux valgus. $P^{* * *}$ : comparisons between mild and severe hallux valgus

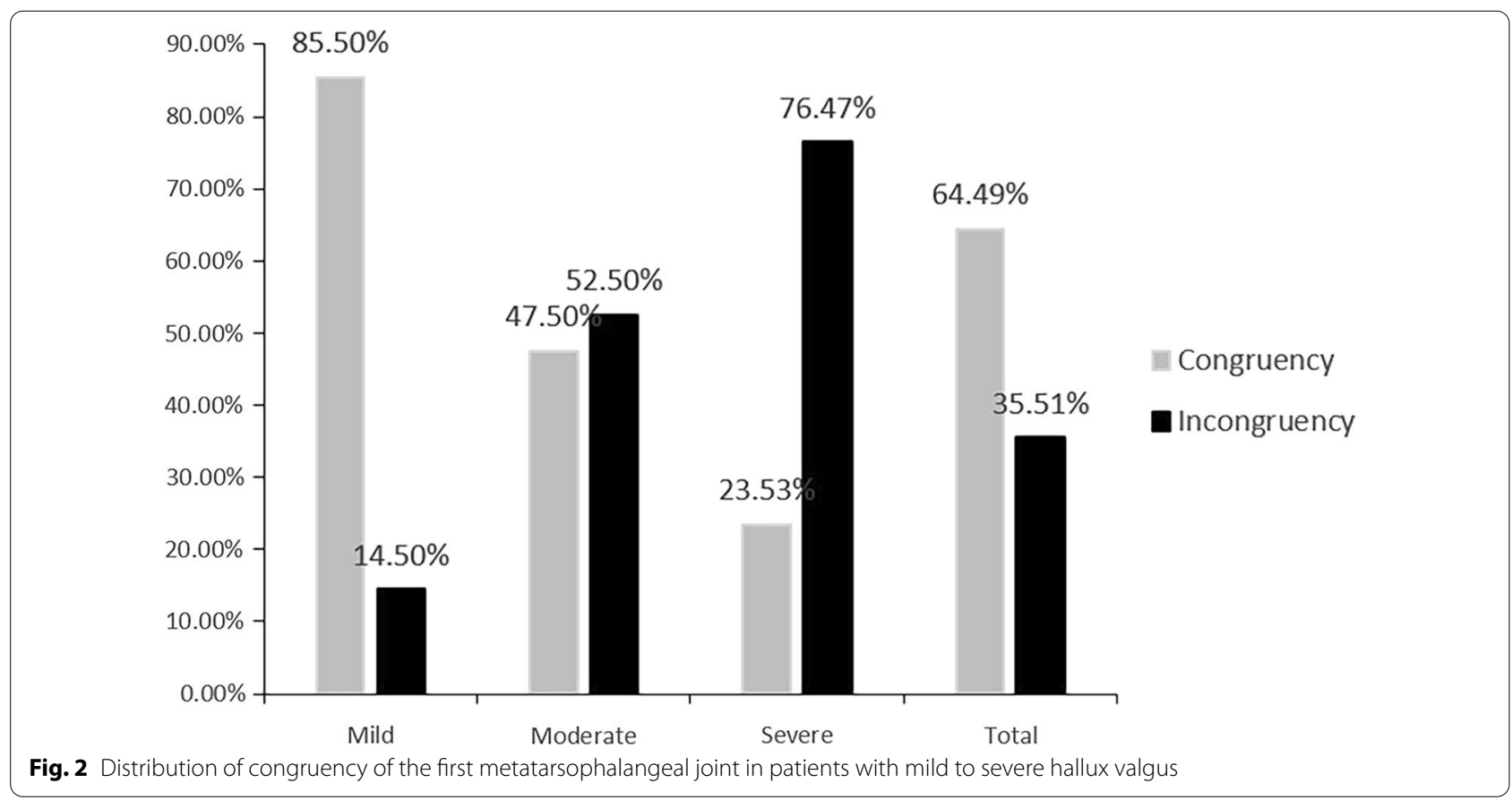

DMAA, the mild patients had no significant difference between the congruency and incongruency groups, but for moderate-to-severe hallux valgus, the DMAA of the congruency group was greater than that of the incongruency group. Among all hallux valgus patients, the MPJA of congruency group was significantly lower than that of incongruency group, while the CI was significantly higher (Table 2 ).

Because of the significant difference in the DMAA, MTPJA and CI between the two groups of patients with moderate-to-severe hallux valgus $(P<0.001)$, we performed diagnostic tests and plotted the ROC curve with the DMAA, MTPJA and CI data. The AUC of the ROC curve for DMAA was $0.554(P>0.05)$ (Fig. 3). However, the MTPJA and CI were 0.906 and 0.884 , respectively, which were both greater than $0.7(P<0.001)$ and showed significant diagnostic value (Figs. 4, 5). The sensitivity and specificity of the MTPJA reached 0.791 and 0.862 , respectively, and the sensitivity and specificity of the CI reached 0.949 and 0.644 . In addition, the critical value of the MTPJA was 10.67, and that of the CI was 0.765 (Table 3).

Correlation tests were performed on 5 parameters between the two groups of patients. In the congruency 
Table 2 Comparison and analysis of different index of patients with different degrees of hallux valgus between congruency and incongruency groups

\begin{tabular}{|c|c|c|c|c|c|}
\hline Groups & Index & Congruency & Incongruency & $T$ value & $P$ value \\
\hline \multirow[t]{5}{*}{ Mild } & $\mathrm{HVA}\left({ }^{\circ}\right)$ & $23.60 \pm 4.00$ & $26.78 \pm 2.88$ & -4.172 & 0.000 \\
\hline & $\operatorname{MTPJA}\left({ }^{\circ}\right)$ & $7.41 \pm 3.99$ & $13.23 \pm 6.58$ & -3.744 & 0.001 \\
\hline & $\operatorname{DMAA}\left({ }^{\circ}\right)$ & $11.27 \pm 5.69$ & $14.20 \pm 6.22$ & -1.350 & 0.179 \\
\hline & $\mathrm{Cl}$ & $0.86 \pm 0.06$ & $0.78 \pm 0.07$ & 5.071 & 0.000 \\
\hline & TSP & $3.55 \pm 1.55$ & $5.74 \pm 0.81$ & -9.265 & 0.000 \\
\hline \multirow[t]{5}{*}{ Moderate } & $\mathrm{HVA}\left({ }^{\circ}\right)$ & $32.98 \pm 2.54$ & $34.39 \pm 2.86$ & -2.329 & 0.022 \\
\hline & $\operatorname{MTPJA}\left({ }^{\circ}\right)$ & $7.57 \pm 4.72$ & $17.73 \pm 6.05$ & -8.309 & 0.000 \\
\hline & $\operatorname{DMAA}\left({ }^{\circ}\right)$ & $22.04 \pm 7.77$ & $16.22 \pm 5.54$ & 3.888 & 0.000 \\
\hline & $\mathrm{Cl}$ & $0.85 \pm 0.06$ & $0.75 \pm 0.08$ & 6.932 & 0.000 \\
\hline & TSP & $4.37 \pm 1.85$ & $5.86 \pm 1.09$ & -4.320 & 0.000 \\
\hline \multirow[t]{5}{*}{ Severe } & $\mathrm{HVA}\left({ }^{\circ}\right)$ & $43.98 \pm 1.89$ & $47.30 \pm 5.06$ & -1.803 & 0.081 \\
\hline & $\operatorname{MTPJA}\left({ }^{\circ}\right)$ & $4.90 \pm 3.32$ & $29.12 \pm 10.42$ & -6.415 & 0.000 \\
\hline & $\operatorname{DMAA}\left({ }^{\circ}\right)$ & $32.45 \pm 5.04$ & $18.79 \pm 10.00$ & 5.154 & 0.000 \\
\hline & $\mathrm{Cl}$ & $0.80 \pm 0.05$ & $0.61 \pm 0.15$ & 5.925 & 0.000 \\
\hline & TSP & $5.50 \pm 1.41$ & $6.27 \pm 0.92$ & -1.447 & 0.182 \\
\hline
\end{tabular}

HVA hallux valgus angle, DMAA distal metatarsal articular angle, MTPJA the first metatarsophalangeal joint angle, $\mathrm{Cl}$ congruency index, TSP tibial sesamoid position
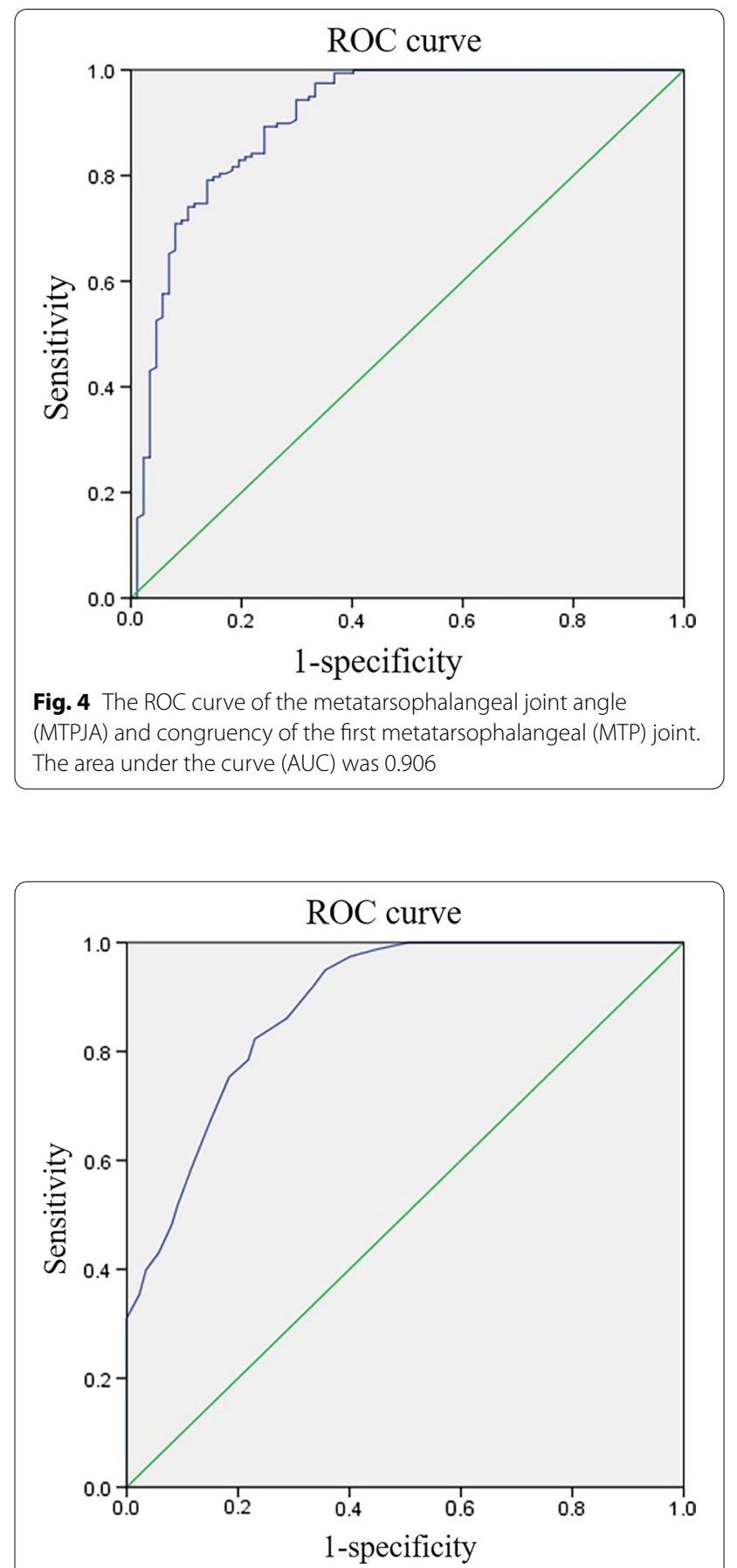

Fig. 5 The ROC curve of the congruency index (Cl) and congruency of the first metatarsophalangeal (MTP) joint. The area under the curve (AUC) was 0.884

Fig. 3 The ROC curve of DMAA and congruency of the first
metatarsophalangeal (MTP) joint. The area under the curve (AUC) was 0.554

group, the DMAA was positively correlated with the HVA, while the correlation coefficients between the MTPJA, CI and HVA were low. In the incongruency group, the DMAA was not correlated with the HVA, while the MTPJA was positively correlated with the HVA,

and the CI was negatively correlated with the HVA. The MTPJA and CI were negatively correlated in both the congruency group and the incongruency group (Table 4). 
Table 3 Diagnostic test between two three index and congruency of the metatarsophalangeal joint

\begin{tabular}{|c|c|c|c|c|c|c|c|c|c|c|}
\hline Index & Critical value & Sensitivity & Specificity & AUC & $P$ value & Youden index & PPV & NPV & $+\mathrm{LR}$ & $-\mathrm{LR}$ \\
\hline DMAA & 12.125 & 0.399 & 0.747 & 0.554 & 0.163 & 0.146 & 0.594 & 0.259 & 1.577 & 0.534 \\
\hline MTPJA & 10.670 & 0.791 & 0.862 & 0.906 & 0.000 & 0.653 & 0.912 & 0.694 & 5.736 & 0.242 \\
\hline $\mathrm{Cl}$ & 0.765 & 0.949 & 0.644 & 0.884 & 0.000 & 0.593 & 0.829 & 0.875 & 2.664 & 0.079 \\
\hline
\end{tabular}

$D M A A$ distal metatarsal articular angle, MTPJA metatarsophalangeal joint angle, $C l$ congruency index, $A U C$ area under the curve, $P P V$ positive predictive value, NPV negative predictive value, $+L R$ positive likelihood ratio, $-L R$ negative likelihood ratio

Table 4 Correlation test of different index between two groups

\begin{tabular}{|c|c|c|c|c|}
\hline Index & MTPJA & DMAA & $\mathrm{Cl}$ & TSP \\
\hline \multicolumn{5}{|c|}{ Congruency group } \\
\hline HVA & $0.103(0.197)$ & $0.691(0.000)$ & $-0.272(0.001)$ & $0.345(0.000)$ \\
\hline MTPJA & - & $-0.377(0.000)$ & $-0.538(0.000)$ & $0.036(0.654)$ \\
\hline DMAA & - & - & $0.057(0.475)$ & $0.517(0.000)$ \\
\hline $\mathrm{Cl}$ & - & - & - & $-0.392(0.000)$ \\
\hline \multicolumn{5}{|c|}{ Incongruency group } \\
\hline HVA & $0.554(0.000)$ & $0.212(0.048)$ & $-0.546(0.000)$ & $0.331(0.002)$ \\
\hline MTPJA & - & $-0.468(0.000)$ & $-0.760(0.000)$ & $0.389(0.000)$ \\
\hline DMAA & - & - & $0.322(0.002)$ & $-0.101(0.350)$ \\
\hline $\mathrm{Cl}$ & - & - & - & $-0.557(0.000)$ \\
\hline
\end{tabular}

HVA hallux valgus angle, DMAA distal metatarsal articular angle, MTPJA the first metatarsophalangeal joint angle, $\mathrm{Cl}$ congruency index, TSP tibial sesamoid position

\section{Discussion}

The congruency of the first MTP joint plays an important role in the selection of the surgical method for post-operative recurrence in hallux valgus. Studies have shown that incongruency of the post-operative MTP joint was highly correlated with the recurrence of hallux valgus [11]. The previous literature reported [11] that the congruency of the first MTP joint has been evaluated only by a doctor's visual assessment of whether the arcs of the MTP joint articular surface were parallel; however, there is no quantitative indicator. In addition, the DMAA is often used to assess dislocation of the first MTP joint [14]. For example, for patients with severe hallux valgus accompanied by an increased DMAA, double metatarsal osteotomy (DMO) is an effective surgical method [15]. However, due to the related complications, such as shortening the length of the first metatarsal bone, post-operative avascular necrosis of the metatarsal head and metastatic metatarsalgia, the application of this technique has been limited to a certain extent [16]. Wang's study compared the efficacy of rotating scarf osteotomy and DMO for hallux valgus accompanied by an increased DMAA. It was believed that there was no significant difference between the two methods, but the former had a lower incidence of complications [16]. Jeong [17] used the point-connecting method to measure the HVA and intermetatarsal angle
(IMA) because with the traditional central axis measurement method, it was difficult to reflect the congruency of the MTP joints.

Evidence suggests that the DMAA is not suitable for use in evaluating the congruency of the first MTP joint [18]. For the larger metatarsals of the DMAA, there will also be two situations where the MTP joints are congruent and incongruent. Our statistical results showed that there was an obvious relationship between the patients' DMAA and the congruency of the first MTP joint for moderate-to-severe hallux valgus. The DMAA of the congruency group was significantly larger than that of the incongruency group. The ROC curve results showed that the AUC was only 0.554 and that the $\mathrm{P}$ value was 0.163 , indicating that the use of DMAA cannot accurately assess congruency of the first MTP joint. In terms of the correlation test, the DMAA and HVA were positively correlated in the congruency group, while there was no correlation between the two in the incongruency group. This indicates that if the DMAA is to be used to assess the degree of hallux valgus, it is limited to the congruency group. In patients with significant dislocation of the first MTP joint, the DMAA is less effective in assessing the severity of hallux valgus.

We innovatively proposed two quantitative evaluation indexes for congruency of the first MTP joint on weightbearing foot anterior-posterior images, the MTPJA and CI, which were quantitatively assessed by measuring the angles of the articular surfaces at both ends and the degree of bonding of the articular surfaces (Fig. 1). Because our data were not clinically necessary, many asymptomatic patients were also included, resulting in a larger proportion of patients with mild hallux valgus, most of whom had congruent MTP joints. The proportions of patients with congruency and incongruency with moderate hallux valgus were basically the same. For patients with severe hallux valgus, nearly a quarter of them had congruency of the first MTP joint, which is similar to the data reported by Coughlin [19]. In addition, as the severity of hallux valgus gradually increased, the MTPJA gradually increased, and the CI gradually decreased, indicating that the contact surface of the first MTP joint surface will gradually decrease. 
In comparing the difference between the congruency and incongruency groups, there was no difference in the HVA between the two groups of patients with mild, moderate or severe hallux valgus. However, among patients with moderate-to-severe hallux valgus, the difference between the MTPJA and CI was large; moreover, the $\mathrm{CI}$ of the congruency group was greater than that of the incongruency group, and the MTPJA of the congruency group was smaller than that of the incongruency group, indicating that the MTPJA and CI could be used to effectively assess the congruency of the first MTP joint. ROC curves of the MTPJA and CI showed that the AUCs were 0.906 and 0.884 , respectively, and that both had diagnostic power. The critical value of the MTPJA was 10.67 , and that of the CI was 0.765 . We can thus define the first MTP joint as incongruent if the value of MTPJA is greater than $10^{\circ}$ or $\mathrm{CI}$ is less than 0.765 , and the degree of incongruency can be measured by the specific value of the two. That is, the larger the MTPJA and the smaller the CI, the greater the degree of congruency is. In the congruency group, the correlation coefficients between the MTPJA, CI and HVA were low, while in the incongruency group, the MTPJA and HVA were significantly positively correlated and the CI and HVA were negatively correlated; that is, the more severe the hallux valgus is, the more deviated the normal ranges of the MTPJA and CI are. Of course, the smaller the value of the MTPJA and the larger the value of the CI, the better the matching relationship of hallux valgus is. Therefore, whether in the congruency group or incongruency group, there was a significant negative correlation between the two. In our previous article [6], 36 patients (38 feet) with moderate-to-severe hallux valgus were followed up at different times before and after surgery, and their CI recovered from 0.75 before surgery to 0.95 at the last follow-up. Similar to the HVA, IMA, etc., all of the indicators recovered well, which also verifies the effectiveness of this indicator to a certain extent.

The present paper also has some limitations that should be taken into consideration. First, this study focused only on the statistical analysis of radiological parameters and did not apply the MTPJA and CI to the comparison of parameters before or after the operation in hallux valgus patients. It also did not classify the magnitude of the two parameters relative to the clinical symptoms. This is what we need to include in the next step of our research. In addition, the patients included in this study had a certain deviation. The number of patients with mild hallux valgus was too large, but because metatarsophalangeal joint mismatch mostly occurs in patients with moderate-tosevere hallux valgus, we believe that the data in the study are still reliable.
In summary, in hallux valgus of different degrees, especially in patients with moderate-to-severe hallux valgus, the first MTP joint is either congruent or incongruent. The DMAA has poor performance in evaluating matching relationships, and the previously used imaging indicators are only qualitative evaluations. The MTPJA and $\mathrm{CI}$ can be used to quantitatively evaluate the congruency of the first MTP joint, and $10^{\circ}$ and 0.765 are used as the demarcation points. Clinically, it is necessary to consider the congruency of the first MTP joint in the selection of different degrees of hallux valgus surgery. The MTPJA and $\mathrm{CI}$ can be used as quantitative evaluation indicators.

\section{Acknowledgements}

Not applicable.

\section{Authors' contributions}

$Y L$ was in charge of manuscript writing, clinical data collection and data statistics; XT and KT were in charge of the overall design and acceptance. All authors read and approved the final manuscript.

\section{Funding}

Leading Scientific and Technological Innovation Talents Under the National "Ten Thousand People Plan" (4139Z2B1).

\section{Availability of data and materials}

The datasets used during the current study are available from the corresponding author on reasonable request.

\section{Declarations}

Ethics approval and consent to participate

This study was approved by the Ethics Committee, and the part number was: (B) KY2021052.

\section{Consent for publication}

Not applicable.

\section{Competing interests}

None of the authors have any competing interests to declare.

Received: 11 October 2021 Accepted: 18 February 2022

Published online: 05 March 2022

\section{References}

1. Castioni D, Fanelli D, Gasparini G, et al. Scarf osteotomy for the treatment of moderate to severe hallux valgus: analysis of predictors for midterm outcomes and recurrence. Foot Ankle Surg. 2020;26(4):439-44. https:// doi.org/10.1016/j.fas.2019.05.013.

2. Pentikainen I, Ojala R, Ohtonen P, et al. Preoperative radiological factors correlated to long-term recurrence of hallux valgus following distal chevron osteotomy. Foot Ankle Int. 2014;35(12):1262-7. https://doi.org/ 10.1177/1071100714548703.

3. Deveci A, Firat A, Yilmaz S, et al. Short-term clinical and radiologic results of the scarf osteotomy: what factors contribute to recurrence? J Foot Ankle Surg. 2013;52(6):771-5. https://doi.org/10.1053/j.jfas.2013.04.003.

4. Mathew PG, Sponer P, Pavlata J, et al. Our experience with double metatarsal osteotomy in the treatment of hallux valgus. Acta Medica (Hradec Kralove). 2012;55(1):37-41. https://doi.org/10.14712/18059694.2015.73.

5. Al-Nammari SS, Christofi T, Clark C. Double first metatarsal and akin osteotomy for severe hallux valgus. Foot Ankle Int. 2015;36(10):1215-22. https://doi.org/10.1177/1071100715589173.

6. Li Y, Chen W, Tao X, et al. Clinical effect of metatarsal diaphysis Z-shaped rotating osteotomy in moderate and severe hallux valgus with 
metatarsophalangeal joint incongruency. Zhonghua yi xue za zhi. 2020;100(31):2423-8. https://doi.org/10.3760/cma.j.cn112137-20200 114-00093.

7. Ma Q, Liang X, Lu J. Chevron osteotomy versus scarf osteotomy for hallux valgus correction: a meta-analysis. Foot Ankle Surg Off J Eur Soc Foot Ankle Surg. 2019;25(6):755-60. https://doi.org/10.1016/j.fas.2018.09.003.

8. Kaiser P, Livingston K, Miller PE, et al. Radiographic evaluation of first metatarsal and medial cuneiform morphology in juvenile hallux valgus. Foot Ankle Int. 2018;39(10):1223-8. https://doi.org/10.1177/1071100718 789696.

9. Almalki T, Alatassi R, Alajlan A, et al. Assessment of the efficacy of SERI osteotomy for hallux valgus correction. J Orthop Surg Res. 2019. https:// doi.org/10.1186/s13018-019-1067-3.

10. Tonogai I, Sairyo K. Temporary Kirschner wire fixation of the first metatarsophalangeal joint before osteotomy for hallux valgus[J]. Int J Surg Case Rep. 2021;84:106104. https://doi.org/10.1016/j.ijscr.2021.106104.

11. Fuhrmann RA, Zollinger-Kies $\mathrm{H}$, Kundert $\mathrm{H}$. Mid-term results of Scarf osteotomy in hallux valgus. Int Orthop. 2010;34(7):981-9. https://doi.org/ 10.1007/s00264-010-0958-z.

12. Mann RA, Saltzman CL. Surgery of the foot and ankle. 2nd ed. Beijing: People's Medical Publishing House; 2014. p. 129.

13. Coughlin MJ, Jones CP. Hallux valgus: demographics, etiology, and radiographic assessment. Foot Ankle Int. 2007;28(7):759-77. https://doi.org/10. 3113/FAl.2007.0759.

14. Robinson AH, Cullen NP, Chhaya NC, et al. Variation of the distal metatarsal articular angle with axial rotation and inclination of the first metatarsal. Foot Ankle Int. 2006;27(12):1036-40. https://doi.org/10.1177/ 107110070602701207.

15. Park CH, Lee WC. Is double metatarsal osteotomy superior to proximal chevron osteotomy in treatment of hallux valgus with increased distal metatarsal articular angle? J Foot Ankle Surg. 2018;57(2):241-6. https:// doi.org/10.1053/j.jas.2017.08.020.

16. Wang X, Wen Q, Li Y, et al. Introduction the revolving scarf osteotomy for treating severe hallux valgus with an increased distal metatarsal articular angle: a retrospective cohort study. BMC Musculoskelet Disord. 2019;20(1):508. https://doi.org/10.1186/s12891-019-2874-8.

17. Seo J, Ahn J, Boedijono D. Point-connecting measurements of the hallux valgus deformity: a new measurement and its clinical application. Yonsei Med J. 2016;57(3):741. https://doi.org/10.3349/ymj.2016.57.3.741.

18. Frumberg DB, Naziri Q, Pivec $R$, et al. Rotational deformity of the first ray precludes accurate distal metatarsal articular angle measurement in hallux valgus. J Long-Term Eff Med Implants. 2018;28(1):41-6. https://doi. org/10.1615/JLongTermEffMedlmplants.2017020615.

19. Coughlin MJ. Hallux valgus in men: effect of the distal metatarsal articular angle on hallux valgus correction. Foot Ankle Int. 1997;18(8):463-70. https://doi.org/10.1177/107110079701800802.

\section{Publisher's Note}

Springer Nature remains neutral with regard to jurisdictional claims in published maps and institutional affiliations.

Ready to submit your research? Choose BMC and benefit from:

- fast, convenient online submission

- thorough peer review by experienced researchers in your field

- rapid publication on acceptance

- support for research data, including large and complex data types

- gold Open Access which fosters wider collaboration and increased citations

- maximum visibility for your research: over $100 \mathrm{M}$ website views per year

At BMC, research is always in progress.

Learn more biomedcentral.com/submissions 